\title{
Some Properties of Legendre Functions and Related Applications
}

\author{
Chie-Ping Chu
}

\begin{abstract}
A bstract. Some properties of Legendre functions in an asymmetric interval (with respect to zero) with zero boundary values are obtained through variational methods. There are given some applications to the monotonicity and estimates of the first Dirichlet eigenvalue for moving bands on $S^{2}$.
\end{abstract}

Keywords: Peak and shape of Legendre functions, monotonicity and estimates of eigenvalues AMS subject classification: $34 \mathrm{~B} 30,34 \mathrm{C} 11,35 \mathrm{P} 15,49 \mathrm{R} 05$

\section{Introduction}

Legendre functions are a classical topic, still their behavior over the interval between two consecutive zeros does not seem to have been thoroughly investigated. For $h \in(0,1)$ and $a$ such that $-\frac{1}{2} h \leq a<1-h$ we consider the Dirichlet eigenvaluc problem for the Legendre equation in $(a, a+h)$

$$
\left.\begin{array}{r}
\frac{d}{d z}\left[\left(1-z^{2}\right) \frac{d F}{d z}\right]+\lambda F(z)=0 \\
F(a)=F(a+h)=0
\end{array}\right\} .
$$

Let $u_{a}>0$ denote the first eigenfunction of problem (1) corresponding to the first cigenvalue $\lambda=\lambda_{a}>0$. Then according to the variational principle (see [1:p.104])

$$
\lambda_{a}=\inf _{F \in H_{a}} \frac{\int_{a}^{a+h}\left(1-z^{2}\right)\left[F^{\prime}(z)\right]^{2} d z}{\int_{a}^{a+h} F^{2}(z) d z}
$$

where $H_{a}=W_{0}^{1,2}(a, a+h)$ is the standard Sobolev space. With sorne variational method, first we estimate the position of the "peak" of $u_{a}$ as well as get some quantitative results about the shape of $u_{a}$ (see Theorem 1). Also, we prove that $\lambda_{a}$ is a decreasing function of $a$ (see Theorem 2 ) which leads to some monotonicity property of the distance between two consecutive zeros of any Legendre function (see Corollary 1).

As a type of special functions, Legendre functions are useful in applications. Among those we mention for example [8] where it is proved by differentiation through spherical

Chie-Ping Chu: Soochow University, Dept. Math., Shihlin, Taipei, Taiwan 11102.R.O.C.

chieping@math.scu.edu.tw 
coordinates that among all equal-area spherical bands the first Dirichlet eigenvalue decreases as the band moves toward the north polc. If we treat these equal-area spherical bands through cylindrical coordinates (we think that for equal-area spherical bands it is natural to use this coordinate system), then the Legendre equation would appear. It follows that we could obtain not only the monotonicity of those first eigenvalues as a corollary of Theorem 2, but wc also give some estimates for those smallest eigenvalues. In particular, we could locate (sharp in certain cases) the first Dirichlet eigenvalue of the equal-area spherical cap (which is the band containing the north pole, see Section 3 ) between $n(n+1)$ and $(n+1)(n+2)$ with positive integer $n$ depending only on the area (see Theorem 3 ). It turns out that we can get a universal lower bound depending only on the area of the above mentioned first eigenvalues. Moreover, by the same technique, we could also get similar results for the first Dirichlet eigenvalues on general surfaces of revolution (Section 4 ).

\section{Main results}

Denote the (unique) point in $[a, a+h]$ which attains the maximum value of $u_{a}(z)$ by $z_{0}$. Then first we will give some information about the position of $z_{0}$ and the shape of $u_{a}$ :

\section{Theorem 1.}

(i) $a+\frac{1}{2} h<z_{0} \leq M$ for $a \in\left(-\frac{1}{2} h, 1-h\right)$ where $M \stackrel{1}{=}\left[\frac{a^{\alpha}+(a+h)^{\alpha}}{2}\right]^{1 / \alpha}$ for $a \in[0,1-h)$ and $M=\frac{1}{2}^{1 / \alpha}(a+h)$ for $a \in\left(-\frac{1}{2} h, 0\right)$, with $\alpha>1$ satisfying $(a+h)^{2} \leq \frac{\alpha-1}{\alpha+1}$.

(ii) For $a \in\left[-\frac{1}{2} h, 1+h\right), u_{a}(z) \leq u_{a}\left(z^{*}\right)$ for $z \in\left[a, a+\frac{1}{2} h\right]$ where $z^{*}$ is the reflection point of $z$ with respect to $z=a+\frac{1}{2} h$.

(iii) For $\left.a \in \frac{1}{2} h, 0\right), u^{\prime}(z)>\left|u^{\prime}(-z)\right|$ for $z \in(a, 0)$.

(iv) For $a \in\left[-\frac{1}{2} h, 1-h\right), u_{a}^{\prime}(a) \leq-u_{a}^{\prime}(a+h)=\left|u_{a}^{\prime}(a+h)\right|$.

Proof. Fix $a \in\left[-\frac{1}{2} h, 1-h\right)$. For convenience, we write $\lambda$ for $\lambda_{a}$ and $u(z)$ for $u_{a}(z)$ here. Consider $w(z)=u(-z)$ for $z \in[-a-h,-a]$. Then

$$
\begin{aligned}
{\left[\left(1-z^{2}\right) w^{\prime}\right]^{\prime}(z)+\lambda w(z) } & =0 \text { in }(-a-h,-a) \\
w(-a-h)=w(-a) & =0 .
\end{aligned}
$$

We will complete the proof of Theorem 1 through the folling Lemmas $1-5$.

Lemma 1. For $a \in\left[-\frac{1}{2} h, 1-h\right), u^{\prime}(z)>0$ in $\left(a, z_{0}\right), u^{\prime}(z)<0$ in $\left(z_{0}, a+h\right)$ and $u^{\prime \prime}(z)<0$ in $(a, a+h)$.

Proof. Since $\left[\left(1-z^{2}\right) u^{\prime}\right]^{\prime}(z)=-\lambda u(z)<0$, we know that $\left(1-z^{2}\right) u^{\prime}(z)$ is strictly decreasing in $(a, a+h)$ which implies that $u^{\prime}(z)>0$ in $\left(a, z_{0}\right)$ and $u^{\prime}(z)<0$ in $\left(z_{0}, a+h\right)$ by the fact that $u^{\prime}\left(z_{0}\right)=0$. To prove that $u^{\prime \prime}(z)<0$ in $(a, a+h)$, first we consider the case $a<0$. We see that in $(a, 0)$ the increasing of $1-z^{2}$ implies that $u^{\prime}(z)$ must be decreasing, hence $u^{\prime \prime}(z)<0$ in $(a, 0)$. Similarly we could know that $w^{\prime \prime}(z)<0$ in $(-a-h, 0)$ from $(2)$ and it follows immediately that $u^{\prime \prime}(z)<0$ in $(0, a+h)$. Besides, a direct computation shows that $u^{\prime \prime}(0)=-\lambda u(0)<0$. In summary, if $a<0$, we have $u^{\prime \prime}(z)<0$ in $(a, a+h)$. As for the case $a \geq 0$, taking account of the increasing of $1-z^{2}$ in $(-a-h,-a)$ we get that $w^{\prime}(z)$ is decreasing hence $w^{\prime \prime}(z)<0$ in $(-a-h,-a)$. It follows that $u^{\prime \prime}(z)<0$ in $(a, a+h)$ 
Lemma 2. $z_{0}>a+\frac{1}{2} h$ for $a \in\left(-\frac{1}{2} h, 1-h\right)$.

Proof. First we reflect $u(z)$ with respect to the line $z=a+\frac{1}{2} h$. Let $z^{*}=2(a+$ $\left.\frac{1}{2} h\right)-z$ be the reflection point of $z$ with respect to $z=a+\frac{1}{2} h$ and define $t(z)=u\left(z^{*}\right)$ for $z \in[a, a+h]$. Then

$$
\left[1-\left(z^{*}\right)^{2}\right] t^{\prime \prime}(z)+2 z^{*} t^{\prime}(z)+\lambda t(z)=0
$$

or

$$
\frac{d}{d z}\left\{\left[1-\left(z^{*}\right)^{2}\right] \frac{d t}{d z}\right\}+\lambda t(z)=0
$$

in $(a, a+h)$ with $t(a)=t(a+h)=0$. Now that $u(z)$ satisfies (1) would imply

$$
\begin{gathered}
\int_{a}^{a+\frac{1}{2} h}\left(1-z^{2}\right)\left[u^{\prime}(z)\right]^{2} d z-\left[1-\left(a+\frac{1}{2} h\right)^{2}\right] u^{\prime}\left(a+\frac{1}{2} h\right) u\left(a+\frac{1}{2} h\right) \\
=\lambda \int_{a}^{a+\frac{1}{2} h} u^{2}(z) d z
\end{gathered}
$$

while $\left(3^{\prime}\right)$ and $t(a+h)=0$ imply

$$
\begin{gathered}
\int_{a+\frac{1}{2} h}^{a+h}\left[1-\left(z^{*}\right)^{2}\right]\left[t^{\prime}(z)\right]^{2} d z+\left[1-\left(a+\frac{1}{2} h\right)^{2}\right] t^{\prime}\left(a+\frac{1}{2} h\right) t\left(a+\frac{1}{2} h\right) \\
=\lambda \int_{a+\frac{1}{2} h}^{a+h} t^{2}(z) d z .
\end{gathered}
$$

Suppose $z_{0} \leq a+\frac{1}{2} h$. Then $u^{\prime}\left(a+\frac{1}{2} h\right) \leq 0$ and accordingly $t^{\prime}\left(a+\frac{1}{2} h \geq 0\right.$. It follows that

$$
\int_{a}^{a+\frac{1}{2} h}\left(1-z^{2}\right)\left[u^{\prime}(z)\right]^{2} d z \leq \lambda \int_{a}^{a+\frac{1}{2} h} u^{2}(z) d z
$$

and

$$
\int_{a+\frac{1}{2} h}^{a+h}\left(1-z^{2}\right)\left[t^{\prime}(z)\right]^{2} d z<\int_{a+\frac{1}{2} h}^{a+h}\left[1-\left(z^{*}\right)^{2}\right]\left[t^{\prime}(z)\right]^{2} d z \leq \lambda \int_{a+\frac{1}{2} h}^{a+h} t^{2}(z) d z .
$$

Since $u\left(a+\frac{1}{2} h\right)=t\left(a+\frac{1}{2} h\right)$, so the function $k$ defined by $k(z)=u(z)$ for $z \in\left[a, a+\frac{1}{2} h\right]$ and $k(z)=t(z)$ for $z \in\left[a+\frac{1}{2} h, a+h\right]$ is in $H_{\mathrm{a}}$. But from (4) - (5) we get

$$
\frac{\int_{a}^{a+h}\left(1-z^{2}\right)\left[k^{\prime}(z)\right]^{2} d z}{\int_{a}^{a+h} k^{2}(z) d z}<\lambda
$$

which contradicts the variational principle. Hence $z_{0}>a+\frac{1}{2} h$ 
Lemma 3. When $a \geq 0$, then $z_{0} \leq \frac{\sqrt[0]{a^{\alpha}+(a+h)^{\alpha}}}{\sqrt[a]{2}}$ and when $a<0$, then $z_{0} \leq \frac{a+h}{\sqrt[a]{2}}$ where $\alpha>1$ satisfies $(a+h)^{2} \leq \frac{\alpha-1}{\alpha+1}$.

Proof. First we consider the case $a \geq 0$. For $z \in(a, a+h)$ let $x=z^{\alpha}$ where $\alpha>1$ satisfies $(a+h)^{2} \leq \frac{\alpha-1}{\alpha+1}$. Then for $x \in\left(a^{\alpha},(a+h)^{\alpha}\right)$, from $(1), u(z(x))$ would satisfy

$$
\alpha^{2} x^{2-(2 / \alpha)}\left(1-x^{2 / \alpha}\right) u^{\prime \prime}(x)+\alpha x\left[(\alpha-1) x^{-(2 / \alpha)}-(\alpha+1)\right] u^{\prime}(x)+\lambda u(x)=0
$$

with $u(x)=0$ at $x=(a+h)^{\alpha}$. Observe that the cocfficient of $u^{\prime}(x)$ in $\left(1^{\prime}\right)$ is nonnegative. From [3: Theorem 2.1] it reveals that $u^{\prime}(x)<0$ for $x \in\left(\frac{a^{\alpha}+(a+h)^{\alpha}}{2},(a+h)^{\alpha}\right)$. It follows that $u(z)$ is decreasing from $z=\left(\frac{a^{\alpha}+(a+h)^{a}}{2}\right)^{1 / \alpha}$ straightforward to $z=a+h$.

Secondly, consider the case $a<0$. We only transform (1) as in the previous case for $z \in(0, a+h)$ to $\left(1^{\prime}\right)$ for $x \in\left(0,(a+h)^{\alpha}\right)$. Then we could know that $u^{\prime}(x)<0$ in $\left(\frac{(a+h)^{\alpha}}{2},(a+h)^{\alpha}\right)$ and to get that $u(z)$ is decreasing from $z=\frac{a+h}{\sqrt[a]{2}}$ straightforward to $z=a+h$

Lemma 4. For each $a \in\left[-\frac{1}{2} h, 1-h\right)$ we have:

(i) $u^{\prime}(a) \leq-u^{\prime}(a+h)$.

(ii) $u(z) \leq u\left(z^{*}\right)$ for $z \in\left(a, a+\frac{1}{2} h\right)$ where $z^{*}$ is the reflection point of $z$ with respect to $z=a+\frac{1}{2} h$.

Proof. Let $t(z)$ be defined as in the proof of Lemma 2. We also define

$$
\xi(z)=u(z)-t(z)=u(z)-u\left(z^{*}\right) \quad \text { for } z \in[a, a+h] .
$$

Then

$$
\left.\begin{array}{rl}
\xi(z) & \equiv-\xi\left(z^{*}\right) \\
\xi^{\prime}(z) & \equiv-\xi^{\prime}\left(z^{*}\right)
\end{array}\right\}
$$

and for $z \in(a, a+h)$ it satisfies

$$
\left(1-z^{2}\right) \xi^{\prime \prime}(z)-2 z \xi^{\prime}(z)+\lambda \xi(z)=4\left(a+\frac{1}{2} h\right)\left\{\left[z-\left(a+\frac{1}{2} h\right) \mid t^{\prime \prime}(z)+t^{\prime}(z)\right\}\right.
$$

with $\xi(a)=\xi(a+h)=0$. Since $z_{0}>a+\frac{1}{2} h$, so $z_{0}^{*}<z_{0}$ and $u(z)$ is increasing for $z \in\left[z_{0}^{*}, z_{0}\right]$. Hence $\xi(z)=u(z)-u\left(z^{*}\right)<0$ and $\xi^{\prime}(z)=u^{\prime}(z)+u^{\prime}\left(z^{*}\right)>0$ for $z \in\left[z_{0}^{*}, a+\frac{1}{2} h\right]$. We claim that $\xi(z) \leq 0$ for $z \in\left(a, z_{0}^{*}\right)$. Then we would have $\xi^{\prime}(a) \leq 0$ by the fact that $\xi(a)=0$. In fact, suppose there were a point $z_{1} \in\left(a, z_{0}^{*}\right)$ and a constant $\delta>0$ such that $\xi\left(z_{1}\right)=0$ as well as $\xi(z)>0$ for $z \in\left(z_{1}-\delta, z_{1}\right) \subseteq\left(a, z_{1}\right)$ and $\xi(z)<0$ for $z \in\left(z_{1}, z_{0}^{*}\right)$. Then $\xi^{\prime}\left(z_{1}\right) \leq 0$. These yield

$$
\begin{array}{r}
u\left(z_{1}^{*}\right)-t\left(z_{1}^{*}\right)=\xi\left(z_{1}^{*}\right)=-\xi\left(z_{1}\right)=0 . \\
u^{\prime}\left(z_{1}^{*}\right)-t^{\prime}\left(z_{1}^{*}\right)=\xi^{\prime}\left(z_{1}^{*}\right)=\xi^{\prime}\left(z_{1}\right) \leq 0 .
\end{array}
$$

Observe that

$$
\begin{aligned}
\int_{z_{i}}^{a+h} & \left(1-z^{2}\right)\left[t^{\prime}(z)\right]^{2} d z-\int_{z_{i}^{*}}^{a+h}\left[1-\left(z^{*}\right)^{2}\right]\left[t^{\prime}(z)\right]^{2} d z \\
\quad= & \int_{z_{i}^{*}}^{a+h}\left[\left(z^{*}\right)^{2}-z^{2}\right]\left[t^{\prime}(z)\right]^{2} d z \\
= & -\int_{z_{i}}^{a+h} t(z) \frac{d}{d z}\left\{\left[z^{2}-\left(z^{*}\right)^{2}\right]\left[-t^{\prime}(z)\right]\right\} d z-\left\{\left[z_{1}^{2}-\left(z_{1}^{*}\right)^{2}\right] t^{\prime}\left(z_{1}^{*}\right)\right\} t\left(z_{1}^{*}\right) .
\end{aligned}
$$


Also, that $u(z)$ satisfies (1) would yield

$$
\int_{a}^{z_{i}^{i}}\left(1-z^{2}\right)\left[u^{\prime}(z)\right]^{2} d z=\lambda \int_{a}^{z_{i}^{*}} u^{2}(z) d z+\left[1-\left(z_{1}^{*}\right)^{2}\right] u^{\prime}\left(z_{1}^{*}\right) u\left(z_{1}^{*}\right) .
$$

Moreover, from $\left(3^{\prime}\right)$ and $t(a+h)=0$ we have

$$
\int_{z_{i}^{*}}^{a+h}\left[1-\left(z^{*}\right)^{2}\right]\left[t^{\prime}(z)\right]^{2} d z=\lambda \int_{z_{i}^{*}}^{a+h} t^{2}(z) d z-\left(1-z_{1}^{2}\right) t^{\prime}\left(z_{1}^{*}\right) t\left(z_{1}^{*}\right) .
$$

Adding the three identities together and taking into account $u\left(z_{1}^{*}\right)=t\left(z_{1}^{*}\right)$ we get

$$
\begin{aligned}
\int_{a}^{z_{i}^{*}}(1 & \left.-z^{2}\right)\left[u^{\prime}(z)\right]^{2} d z+\int_{z_{i}^{*}}^{a+h}\left(1-z^{2}\right)\left[t^{\prime}(z)\right]^{2} d z \\
= & \lambda\left[\int_{a}^{z_{i}^{*}} u^{2}(z) d z+\int_{z_{i}^{*}}^{a+h} t^{2}(z) d z\right] \\
& -\int_{z_{i}^{*}}^{a+h} t(z) \frac{d}{d z}\left\{\left[z^{2}-\left(z^{*}\right)^{2}\right]\left[-t^{\prime}(z)\right]\right\} d z \\
+ & {\left[1-\left(z_{1}^{*}\right)^{2}\right]\left[u^{\prime}\left(z_{1}^{*}\right)-t^{\prime}\left(z_{1}^{*}\right)\right] u\left(z_{1}^{*}\right) } \\
< & \lambda\left[\int_{a}^{z_{i}^{*}} u^{2}(z) d z+\int_{z_{i}^{*}}^{a+h} t^{2}(z) d z\right]
\end{aligned}
$$

where the last inequality is due to $u^{\prime}\left(z_{1}^{*}\right) \leq t^{\prime}\left(z_{1}^{*}\right)$ as well as that $\left[z^{2}-\left(z^{*}\right)^{2}\right]$ and $\left[-t^{\prime}(z)\right]$ are both strictly increasing for $z \in\left(z_{1}^{*}, a+h\right)$ (since $z_{1}^{*}>z_{0}>a+\frac{1}{2} h$ ) and that $t(z)$ is positive there. So the integrand of

$$
\int_{z_{i}^{*}}^{a+h} t(z) \frac{d}{d z}\left\{\left[z^{2}-\left(z^{*}\right)^{2}\right]\left[-t^{\prime}(z)\right]\right\} d z
$$

is positive everywhere. Now we define a function $\eta$ in $[a, a+h]$ by $\eta(z)=u(z)$ for $z \in\left[a, z_{1}^{*}\right]$ and $\eta(z)=t(z)$ for $z \in\left[z_{1}^{*}, a+h\right]$ (recall that by assumption $u\left(z_{1}^{*}\right)=t\left(z_{1}^{*}\right)$ ). It follows from (6) that

$$
\frac{\int_{a}^{a+h}\left(1-z^{2}\right)\left[\eta^{\prime}(z)\right]^{2} d z}{\int_{a}^{a+h} \eta^{2}(z) d z}<\lambda
$$

which would raise a contradiction as in the proof of Lemma 2. This proves our claim to be true, that is,

$$
\begin{aligned}
\xi(z)=u(z)-u\left(z^{*}\right) & \leq 0 \text { for } z \in\left(a, a+\frac{1}{2} h\right) \\
\xi^{\prime}(a)=u^{\prime}(a)+u^{\prime}\left(a^{*}\right)=u^{\prime}(a)+u^{\prime}(a+h) & \leq 0
\end{aligned}
$$

and the proof is completed 
Lemma 5. If $a \in\left(-\frac{1}{2} h, 0\right)$, then $u^{\prime}(z)>\left|u^{\prime}(-z)\right|$ for $z \in(a, 0)$.

Proof. For $z \in(a,-a)$, define $v(z)=u(z)-u(-z)$. Then $v$ is an odd function and

$$
\left[\left(1-z^{2}\right) v^{\prime}\right]^{\prime}(z)+\lambda v(z)=0 \quad \text { in }(a,-a) .
$$

Knowing that $z_{0}>a+\frac{1}{2} h>0$, we consider first the case $0<z_{0}<-a$. Since $u^{\prime}(z)>0$ for $z \in\left(-z_{0}, z_{0}\right)$, so $v(z)=u(z)-u(-z)<0$ and $v^{\prime}(z)=u^{\prime}(z)+u^{\prime}(-z)>0$ for $z \in\left(-z_{0}, 0\right)$. Also, a direct computation yields $v^{\prime}\left(-z_{0}\right)=u^{\prime}\left(-z_{0}\right)>0$.

We claim that $v(z)<0$ in $\left(a,-z_{0}\right)$. In fact, if there were a point $z=-z_{1} \in\left(a,-z_{0}\right)$ such that $v\left(-z_{1}\right)=0$, then we would have $u\left(-z_{1}\right)=u\left(z_{1}\right)$. Since the first eigenvalue of problem (1) is non-degencrate, it follows from a standard symmetrization argument that $u(z)$ must be symmetric in $\left(-z_{1}, z_{1}\right)$ and attain the maximum at $z=0$ which induces a contradiction, hence the claim is true. Accordingly, $\left\{\left(1-z^{2}\right) v^{\prime}\right\}^{\prime}(z)=-\lambda v(z)>0$ in $\left(a,-z_{0}\right)$ which implies that $\left(1-z^{2}\right) v^{\prime}(z)$ is increasing in $\left(a,-z_{0}\right)$.

Next we claim that $v^{\prime}(z)>0$ in $\left(a,-z_{0}\right)$. Indeed, if there were a point $z=-z_{2} \epsilon$ $\left(a,-z_{0}\right)$ such that $v^{\prime}\left(-z_{2}\right)=0$ and $v^{\prime}(z)>0$ in $\left(-z_{2},-z_{0}\right)$, let $m(z)=v^{\prime}(z)$ in $\left[-z_{2}, z_{2}\right]$. Then $m$ is even and positive in $\left(-z_{2}, z_{2}\right)$ and it satisfies

$$
\left[\left(1-z^{2}\right) m\right]^{\prime \prime}(z)+\lambda m(z)=0 \text { for } z \in\left(-z_{2}, z_{2}\right) \text { with } m\left(-z_{2}\right)=m\left(z_{2}\right)=0 .
$$

Let $w(z)=\left(1-z^{2}\right) m(z)$ for $z \in\left(-z_{2}, z_{2}\right)$. Then $w(z)>0$ for $z \in\left(0, z_{2}\right)$ and satisfies

$$
w^{\prime \prime}(z)+\frac{\lambda w(z)}{1-z^{2}}=0 \text { for } z \in\left(0, z_{2}\right) \text { with }\left\{\begin{array}{r}
w\left(z_{2}\right)=0 \\
w^{\prime}(0)=m^{\prime}(0)=0
\end{array}\right.
$$

We sce that $(\lambda, w(z))$ is the first. eigenpair for the mixed boundary eigenvalue problem (7). On the other hand, let $n(z)=u^{\prime}(z)$. We define $q(z)=\left(1-z^{2}\right) n(z)$ for $z \in\left(0, z_{0}\right)$ and $q(z)=0$ for $z \in\left(z_{0}, z_{2}\right)$. Then $q\left(z_{0}\right)=0, q$ will be a Lipschitz continuous function in $\left(0, z_{2}\right)$ and it would satisfy the differential equation in $(7)$ for $z \in\left(0, z_{0}\right)$. Since the infimum of the corresponding Rayleigh quotient for $(7)$ in $\left(0, z_{2}\right)$ is attained by $w(z)$ with value being $\lambda$ and the Rayleigh quotient of $q(z)$ over $\left(0, z_{2}\right)$ equals $\lambda$, too (notice that $q(z) \equiv 0$ in $\left.\left[z_{0}, z_{2}\right)\right)$, the variational principle tells that owing to that $\lambda$ is non-degenerate (see [2: p.164]), we would have $q(z) \equiv c w(z)$ in $\left(0, z_{2}\right)$ for some non-zero constant $c$. But $q^{\prime}(0)=u^{\prime}(0) \neq 0=c w^{\prime}(0)$ raises a contradiction. Hence our claim is true, and we have $v^{\prime}(z)>0$ in $\left(a,-z_{0}\right)$. Thus $u^{\prime}(z)>-u^{\prime}(-z)=\left|u^{\prime}(-z)\right|$ for $z \in\left(a,-z_{0}\right)$. Moreover, we know that $u^{\prime}(z)>0$ and is strictly decreasing in $\left(-z_{0}, z_{0}\right)$, hence we also have $u^{\prime}(z)>u^{\prime}(-z)=\left|u^{\prime}(-z)\right|$ for $z \in\left(-z_{0}, 0\right)$.

As for the case $z_{0}>-a$ notice that, now in $(a,-a), u^{\prime}(z)>0$ and is decreasing, it is easy to see the statement to be true

Through Lemmas 1 - 5 we have completed the proof of Theorem 1 . Now we are going to apply Theorem 1 to prove the monotonicity of $\lambda_{a}$ :

Theorem 2. If $a<b$ are both in $\left(-\frac{1}{2} h, 1-h\right)$, then $\lambda_{a}>\lambda_{b}$.

Proof. We write $u$ for $u_{a}$ in this proof for convenience. Set $d=b-a>0$ and define $j(z)=u(z-d)$ for $z \in(b, b+h)$. Then $j \in H_{b}$ (recall that, for $c \in\left[-\frac{1}{2} h, 1-h\right)$, 
$\left.H_{c}=W_{0}^{1,2}(c, c+h)\right)$. We see that $H_{a}$ and $H_{b}$ form a one-one correspondence via this translation. It is trivial that $\int_{a}^{a+h} u^{2}(z) d z=\int_{b}^{b+h} j^{2}(z) d z$. We will show that

$$
\int_{a}^{a+h}\left(1-z^{2}\right)\left[u^{\prime}(z)\right]^{2} d z>\int_{b}^{b+h}\left(1-z^{2}\right)\left[j^{\prime}(z)\right]^{2} d z
$$

if $a<b$. Then the theorem will be true from the variational principle. Let

First consider the case $a<0$. Since $z_{0}>a+\frac{1}{2} h \geq 0$, we have $u^{\prime}(z)>0$ for $z \in(a, 0)$.

$$
A=\left\{z \in(0, a+h):\left|u^{\prime}(z)\right|=u^{\prime}(\sigma) \text { for some } \sigma \dot{\epsilon}(a, 0)\right\} .
$$

Then $\mathrm{A}$ is an open interval contained in $(0, a+h)$. Writc $B=A+d$ to be the set translated from $A$ by the distance $d$. Then we have

$$
\begin{aligned}
\int_{a}^{a+h} & \left(1-z^{2}\right)\left[u^{\prime}(z)\right]^{2} d z-\int_{b}^{b+h}\left(1-\bar{z}^{2}\right)\left[j^{\prime}(z)\right]^{2} d z \\
= & \left(\int_{a}^{0}+\int_{A}+\int_{(0, a+h)-A}\right)\left(1-z^{2}\right)\left[u^{\prime}(z)\right]^{2} d z \\
& -\left(\int_{a+d}^{d}+\int_{B}+\int_{(d, a+h+d)-B}\right)\left(1-z^{2}\right)\left[j^{\prime}(z)\right]^{2} d z \\
= & I+I I \\
> & 0
\end{aligned}
$$

where

$$
\begin{aligned}
I & =\left(\int_{a}^{0}+\int_{A}\right)\left(1-z^{2}\right)\left[u^{\prime}(z)\right]^{2} d z-\left(\int_{a+d}^{d}+\int_{B}\right)\left(1-z^{2}\right)\left[j^{\prime}(z)\right]^{2} d z \\
I I & =\int_{(0, a+h)-A}\left(1-z^{2}\right)\left[u^{\prime}(z)\right]^{2} d z-\int_{(d, d+h)-B}\left(1-z^{2}\right)\left[j^{\prime}(z)\right]^{2} d z .
\end{aligned}
$$

Now we prove that $I>0$. In fact, for each $\sigma \in(a, 0)$ we know that there exists a unique $\tau \in A$ such that $u^{\prime}(\sigma)=\left|u^{\prime}(\tau)\right|=j^{\prime}(\sigma+d)=\left|j^{\prime}(\tau+d)\right|$. Then

$$
\left(\int_{a}^{0}+\int_{A}\right)\left(1-z^{2}\right)\left[u^{\prime}(z)\right]^{2} d z=\int_{a}^{0}\left[\left(1-\sigma^{2}\right)+\left(1-\tau^{2}\right)\right]\left[u^{\prime}(\sigma)\right]^{2} d \sigma
$$

and

$$
\left(\int_{a+d}^{d}+\int_{B}\right)\left(1-z^{2}\right)\left[j^{\prime}(z)\right]^{2} d z=\int_{a}^{0}\left\{\left[1-(\sigma+d)^{2}\right]+\left[1-(\tau+d)^{2}\right]\right\}\left[j^{\prime}(\sigma+d)\right]^{2} d \sigma .
$$

By Theorem 1 we know that $\sigma+\tau>0$ which implies

$$
\begin{aligned}
{[(1-} & \left.\left.\sigma^{2}\right)+\left(1-\tau^{2}\right)\right]\left[u^{\prime}(\sigma)\right]^{2}-\left[1-(\sigma+\tau)^{2}+1-(\tau+d)^{2}\right]\left[j^{\prime}(\sigma+d)\right]^{2} \\
& =\left[u^{\prime}(\sigma)\right]^{2}\left[2 \sigma d+2 \tau d+2 d^{2}\right] \\
& =2 d\left[u^{\prime}(\sigma)\right]^{2}[\sigma+\tau+d] \\
& >0
\end{aligned}
$$


for each $d>0$. Hence $I>0$.

Next we prove that $I I>0$. In fact, for $z \in(0, a+h)-A,\left(1-z^{2}\right)$ is decreasing, so

$$
\begin{aligned}
I I & =\int_{(0, a+h)-A}\left(1-z^{2}\right)\left[u^{\prime}(z)\right]^{2} d z-\int_{(0, a+h)-A}\left[1-(z+d)^{2}\right]\left[j^{\prime}(z+d)\right]^{2} d z \\
& =\int_{(0, a+h)-A}\left\{\left(1-z^{2}\right)-\left[1-(z+d)^{2}\right]\right\}\left[u^{\prime}(z)\right]^{2} d z \\
& >0 .
\end{aligned}
$$

Sccondly, consider the case $a>0$. Since $\left(1-z^{2}\right)$ is decreasing in $(a, a+h)$, the result is casily seen to be true as the previous computation in the proof of $I I>0$

The technique of proving $I I>0$ in Theorem 2 could be used to get some generalization of Theorem 2. Also the monotonicity of $\lambda_{a}$ reveals that the distance betwcen two consecutive zeros of any Legendre function would be shorter as the zeros get larger. We have the following

Corollary 1. Let $\mu_{a}$ be the first eigenvalue of the Dirichlet-Sturm-Liouville operator $\frac{d}{d z}\left[p(z) \frac{d}{d z}\right]$ over $(a, a+h)$ with $p$ being positive and continuous in $[a, a+h]$. If $p$ is decreasing in $(a, a+h)$, then $\mu_{\mathbf{a}}$ will decrease with respect to $a$.

Corollary 2. Suppose $p_{v}$ is a solution of the differential equation in (1) over $(-1,1)$ with $v(v+1)=\lambda$, and $y_{1}<y_{2}<y_{3}$ are consecutive zeros of $p_{v}$ with $y_{1} \geq y_{0}$ where $y_{0}$ is the largest non-positive zero of $p_{v}$. Then $y_{3}-y_{2}<y_{2}-y_{1}$.

Proof. If $y_{3}-y_{2} \geq y_{2}-y_{1}$, then from Theorem 2 and "domain monotonicity" of the first Dirichlet eigenvalues (see [1: p.100]), $p_{v}$ would not satisfy the differential equation in (1) over both $\left(y_{1}, y_{2}\right)$ and $\left(y_{2}, y_{3}\right)$ with the same $\lambda$, which is a contradiction

\section{Applications}

Denote by $S^{2}$ the unit sphere in $\mathbb{R}^{3}$. For $a \in\left[\frac{1}{2} h, 1-h\right)$, let $D_{a}$ be the spherical band on $S^{2}$ parametrized according to cylindrical coordinates:

$$
b(z, \theta)=\left(\left(1-z^{2}\right)^{1 / 2} \cos \theta,\left(1-z^{2}\right)^{1 / 2} \sin \theta, z\right)
$$

for $a<z<a+h$ and $0 \leq \theta<2 \pi$. We see that each $D_{a}$ has the same area $2 \pi h$, that is the equal-area of $D_{a}$ implies the irrelevance of $h$ to $a$. The Beltrami-Laplace operator on $S_{2}$ in our coordinates is written as

$$
\Delta=\frac{\partial}{\partial z}\left[\left(1-z^{2}\right) \frac{\partial}{\partial z}\right]+\frac{\partial}{\partial \theta}\left[\frac{1}{1-z^{2}} \frac{\partial}{\partial \theta}\right]
$$

Due to the non-degeneracy of the first Dirichlet eigenvalue, the corresponding eigenfunction is independent of $\theta$, so $u_{a}(z)$ (in Section 1) is the eigenfunction corresponding to the first eigenvalue $\lambda_{a}$ for the Laplacian in $D_{a}$ with Dirichlet boundary condition. Hence we know: 
Corollary 3. The first Dirichlet eigenvalue is decreasing as the spherical band moved toward the north pole.

Now we will give some estimates of $\lambda_{a}$. First we shall give a lower bound of the first Dirichlet eigenvalue of the spherical cap $D_{0}$ (defined later), which would turn out to be a universal lower bound of all $\lambda_{a}$ for $a \in\left[-\frac{1}{2} h, 1-h\right)$.

Let $D_{0}$ be the spherical cap parametrized by $b(z, \theta)$ for $z \in(1-h, 1]$ and $\theta \in[0,2 \pi)$. As before the first Dirichlet eigenfunction will be independent of $\theta$. Let $\left(\lambda_{0}, u_{0}\right)$ be the first eigenpair for the Laplacian in $D_{0}$ with Dirichlet boundary condition. Then $u_{0}$ would satisfy the differential equation in (1) with the boundary conditions $u_{0}(1-h)=0$ and $u_{0}^{\prime}(1)$ being finite (since $\left.\nabla u(z, \theta)=\left(1-z^{2}\right)^{1 / 2} u^{\prime}(z)\left(-z \cos \theta,-z \sin \theta,\left(1-z^{2}\right)^{1 / 2}\right)\right)$. If we write $\lambda_{0}=v_{0}\left(v_{0}+1\right)$, then $u_{0}=c p_{v_{0}}$ where $c$ is a non-zero constant and $p_{v_{0}}$ is the Legendre function of degree $v_{0}$ of the first kind [5: p.165] with $v_{0}$ being chosen so that $p_{v_{0}}$ has $z=1-h$ to be its largest zero in $(-1,1)$. The other boundary condition is naturally held by $p_{v_{0}}$ since $p_{v_{0}}^{\prime}(z)=\frac{1}{2}(-v)\left(v_{0}+1\right) F\left(1-v_{0}, v_{0}+2,2, \frac{1-z}{2}\right)$ as well as $F\left(1-v_{0}, v_{0}+2,2,0\right)=1$ where $F$ is the herpergeometric series [5: p.193, 197, 238]. Now let $P_{n}$ be the Legendre polynomial of degree $n, z_{n}$ denote the largest (the $n$-th) zero of $P_{n}$ in $(-1,1)$. Then we have

\section{Theorem 3.}

(i) $\lim _{a-1-h} \lambda_{a}=\lambda_{0}$ and $\lambda_{0} \leq \lambda_{a}$ for all $a$ in $\left[-\frac{1}{2} h, 1-h\right)$.

(ii) If $1-h=z_{n}$ for some integer $n>0$, then $\lambda_{0}=n(n+1)$.

(iii) If $z_{n}<1-h<z_{n+1}$, then $n(n+1)<\lambda_{0}<(n+1)(n+2)$.

Proof. By a straight modification of $[7$, p. 551/Lemma $]$ and the decreasing property of $\lambda_{a}$ in Theorem 2, the result of statement (i) is established (also, see [8]). We need only to treat the latter half of the theorem.

It is well known that $P_{n}$ satisfies the differential equation in $(1)$ over $(-1,1)$ with $\lambda=\lambda_{n}=n(n+1)$ and it has exactly $n$ distinct zeros in $(-1,1)$ for $n \in \mathbb{N}$. Also, we could view $\left(\lambda_{n}, P_{n}\right)$ as the first eigenpair of the differential equation in (1) over $\left(z_{n}, 1\right)$ with $P_{n}\left(z_{n}\right)=0, P_{n}^{\prime}(1)$ being finite. If $1-h=z_{n}$ for some $n$, then $u_{0}=c P_{n}$ in $[1-h, 1]$ and $\lambda_{0}=n(n+1)$. On the other hand, from Sturm's Fundamental Theorem we know that $z_{n}$ is increasing as $n$ increases [4: p. 225]. If $1-h \in\left(z_{n}, z_{n+1}\right)$ for some positive integer $n$, then $P_{n+1}$ would be a trial function for the Rayleigh quotient associated with $\lambda_{0}$ over $(1-h, 1]$. Similarly, $u_{0}$ would be a trial function for the Rayleigh quotient associated with $\lambda_{n}=n(n+1)$ over $\left(z_{n}, 1\right]$. Hence we have $n(n+1)<\lambda_{0}<(n+1)(n+2)$. This completes the proof

In [6], $z_{n}$ is tabulated from $n=2$ to $n=16$ :

$$
\begin{aligned}
& z_{2} \cong 0.57735 \\
& z_{3} \cong 0.77460 \\
& z_{4} \cong 0.86114 \\
& z_{5} \cong 0.90618 \\
& z_{6} \cong 0.93247
\end{aligned}
$$$$
z_{7} \cong 0.94911
$$$$
z_{8} \cong 0.96029
$$$$
z_{9} \cong 0.96816
$$$$
z_{10} \cong 0.97390
$$$$
z_{11} \cong 0.97823
$$

$$
\begin{aligned}
& z_{12} \cong 0.98156 \\
& z_{13} \cong 0.98418 \\
& z_{14} \cong 0.98628 \\
& z_{15} \cong 0.98799 \\
& z_{16} \cong 0.98940
\end{aligned}
$$


We observe that when $1-h$ near 1 (that is, a small cap), $\lambda_{0}$ is very sensitive to $h$ (or, to the area of the cap). Also, when $1-h<z_{2}$, the lower bound will not be available. Hence in Theorem 4 we give another form of the lower bound of $\lambda_{a}$ by observing that

$$
\inf _{f \in H_{a}} \frac{\int_{a}^{a+h}\left[f^{\prime}(z)\right]^{2} d z}{\int_{a}^{a+h} f^{2}(z) d z}=\Lambda=\left(\frac{\pi}{h}\right)^{2}
$$

(since it is the first Dirichlet eigenvalue of $f^{\prime \prime}(z)+\Lambda f(z)=0$ in $(a, a+h)$ ). Also, we use the corresponding first eigenfunction $\sin \frac{\pi(z-a)}{h}$ as a trial function of the Rayleigh quotient associated with $\lambda_{a}$ to get an upper bound:

Theorem 4. For $a<0$, we have

$$
1-(a+h)^{2}<\left(\frac{h}{\pi}\right)^{2} \lambda_{a}<1-a(a+h)-\frac{\left(2 \pi^{2}+3\right) h^{2}}{6 \pi^{2}}
$$

and for $a \geq 0$ we have

$$
1-(a+h)^{2}<\left(\frac{h}{\pi}\right)^{2} \lambda_{a}<1-a(a+h)-\frac{\left(2 \pi^{2}+3\right) h^{2}}{6 \pi^{2}}<1-a^{2} .
$$

However, from Theorem 1 we could also give a somewhat improved lower bound. Letting $\alpha$ and $M$ be as in Theorem 1, we have

Corollary 4. $\lambda_{a}>\min \left\{1-M^{2}, 1-a^{2}\right\}\left[\frac{\pi}{2(M-a)}\right]^{2}$.

Proof. Since $(\lambda, u)$ (this is the abbreviation of $\left(\lambda_{a}, u_{a}\right)$ is also the first eigenpair of the differential equation in (1) over $\left(a, z_{0}\right)$ with mixed boundary conditions $u(a)=$ $u^{\prime}\left(z_{0}\right)=0$, we have

$$
\left(\min \left\{1-z_{0}^{2}, 1-a^{2}\right\}\right) \int_{a}^{z_{0}}\left[u^{\prime}(z)\right]^{2} d z<\int_{a}^{z_{0}}\left(1-z^{2}\right)\left[u^{\prime}(z)\right]^{2} d z=\lambda \int_{a}^{z_{0}} u^{2}(z) d z .
$$

Notice that

$$
\Omega=\left[\frac{\pi}{2\left(z_{0}-a\right)}\right]^{2}=\inf _{H} \frac{\int_{a}^{z_{0}}\left[f^{\prime}(z)\right]^{2} d z}{\int_{a}^{z_{0}} f^{2}(z) d z}
$$

(since it is the first eigenvalue of $f^{\prime \prime}(z)+\Omega f(z)=0$ over $\left(a, z_{0}\right)$ with mixed boundary condition $\left.f(a)=f^{\prime}\left(z_{0}\right)=0\right)$, where $H$ is the Sobolev space consisting of functions in $W^{1,2}\left(a, z_{0}\right)$ that vanish at $z=a$. From Lemma 3 we know that $\Omega>\left[\frac{\pi}{2(M-a)}\right]^{2}$. Hence

$$
\lambda_{a}>\left(\min \left\{1-z_{0}^{2}, 1-a^{2}\right\}\right) \Omega>\left(\min \left\{1-M^{2}, 1-a^{2}\right\}\right)\left[\frac{\pi}{2(M-a)}\right]^{2}
$$

and the corollary is proved 


\section{On general surfaces of revolution}

As in Section 3, a general surface of revolution $S$ will be parametrized as $\Phi(z, \theta)=$ $(p(z) \cos \theta, p(z) \sin \theta, z)$ for $0 \leq \theta<2 \pi$ and $z \in I$, with Riemannian measure being $d A=\sqrt{g} d z d \theta, g(z, \theta)=g(z)=p^{2}(z)\left[1+\left(p^{\prime}(z)\right)^{2}\right]$ where $p(z)>0$ is a smooth function in an open interval $I$ on the $z$-axis. We consider the Dirichlet eigenvalue problems for the "equal-area bands" on $S$ :

Let $D_{\mu}$ be a band on $S$ parametrized by $\Phi(z, \theta)$ for $0 \leq \theta<2 \pi$ and $z \in\left(\mu, \mu^{*}\right) \subseteq I$. Then as before, on $D_{\mu}$, the first Dirichlet eigenpair $\left(\lambda_{\mu}, u_{\mu}\right)$ would satisfy

$$
\left(\sqrt{g} \frac{1}{1+\left[\left(p^{\prime}(z)\right]^{2}\right.} u_{\mu}^{\prime}\right)^{\prime}(z)=-\lambda_{\mu} \sqrt{g} u_{\mu}(z) \quad \text { with } u_{\mu}(\mu)=u_{\mu}\left(\mu^{*}\right)=0 .
$$

After changing the variable by $y(z)=\int_{\chi}^{z} \sqrt{g(z)} d z$ with $\chi$ being the left end point of $I$, the area of

$$
D_{\mu}=2 \pi \int_{\mu}^{\mu^{*}} \sqrt{g(z)} d z=2 \pi \int_{c}^{c+k} d y=2 \pi k
$$

with $c=z^{-1}(\mu), c+k=z^{-1}\left(\mu^{*}\right)$ and $u_{\mu}(z(y))$ would satisfy

$$
\frac{d}{d y}\left[p^{2}(y) \frac{d u_{\mu}}{d y}\right]=-\lambda_{\mu} u_{\mu}(y) \quad \text { in }(c, c+k)
$$

with Dirichlet boundary condition. So

$$
\lambda_{\mu}=\inf _{w \in H_{c}} \frac{\int_{c}^{c+k} p^{2}(y)\left[w^{\prime}(y)\right]^{2} d y}{\int_{c}^{c+k} w^{2}(y) d y}
$$

and we could get similar results as in Section 3:

Theorem 5. Let $k=\frac{\text { area } D_{\mu}}{2 \pi}$ as above. Then

$$
\inf _{z \in\left(\mu, \mu^{*}\right)}\left[p^{2}(z)\right]\left(\frac{\pi}{k}\right)^{2} \leq \lambda_{\mu} \leq \sup _{z \in\left(\mu, \mu^{*}\right)}\left[p^{2}(z)\right]\left(\frac{\pi}{k}\right)^{2}
$$

for each $\mu$.

Also, since $y^{\prime}(z)>0$ for $z$ in $I$, we get

Theorem 6. Suppose $p$ is decreasing (increasing respectively) in I. Then $\lambda_{\mu}$ is a decreasing (increasing respectively) function of $\mu$. Moreover, the distance of two consecutive zeros in $I$ would be decreasing (increasing respectively) as the zeros go larger.

Acknowledgment. The auther would like to thank the referee and Prof. $R$. Finn for some constructive suggestions. 


\section{References}

[1] Courant, R. and D. Hilbert: Methods of Mathematical Physics, Vol. 1. New York: Intersci. 1953.

[2] Churchill, R. V. and J. W. Brown: Fourier Series and Boundary Value Problems, 4th ed. Singapore: McGraw-Hill 1987.

[3] Gidas, B., NI, W. M. and L. Nirenberg: Symmetry and related properties vin the maximum principle. Comm. Math. Phys 68 (1979), $209-243$.

[4] Ince, E. L.: Ordinary Differential Equations. New York: Dover Publ. 1926.

[5] Lebedev, N. N.: Special Functions and Their Applications. New Jersey: Prentice-Hall 1965.

[6] Lowan, A. N., Davids, N. and A. Levenson: Table of the Zeros of the Legendre Polynominls of Order 1-16 and the Weight Coefficients for Gauss' Mechanical Quadrature Formula. Bull. Amer. Math. Soc. 48, (1942), 739 - 743.

[7] Osserman, R.: A note on Hayman's theorem on the bass note of a dmum. Comment. Math. Helvetici 52 (1977), 545- 555.

[8] Shen, C. L. and C. T. Shich: Some properties of the first eigenvalue of the Laplace operator on the spherical bands in $S^{2}$. SIAM J. Math. Anal. 23 (1992), 1305-1308. 